\title{
Carba-cyclophellitols Are Neutral Retaining-Glucosidase Inhibitors
}

\author{
Thomas J. M. Beenakker, ${ }^{\dagger}$ Dennis P. A. Wander, ${ }^{\dagger}$ (๖ Wendy A. Offen, ${ }^{\S}$ Marta Artola, ${ }^{\dagger}$ Lluís Raich, $^{\perp}$ \\ Maria J. Ferraz,,$^{\ddagger}$ Kah-Yee Li, ${ }^{\dagger}$ Judith H. P. M. Houben, ${ }^{\ddagger}$ Erwin R. van Rijssel, ${ }^{\dagger}$ Thomas Hansen, ${ }^{\dagger}$ \\ Gijsbert A. van der Marel, ${ }^{\dagger}$ Jeroen D. C. Codée, ${ }^{\dagger}$ Johannes M. F. G. Aerts, ${ }^{\ddagger}$ Carme Rovira, ${ }^{\perp, \|(}$ \\ Gideon J. Davies, ${ }^{*} \S^{\circ}(0)$ and Herman S. Overkleeft ${ }^{*}+{ }^{\dagger}$
}

${ }^{\dagger}$ Department of Bio-organic Synthesis and Department of Medical Biochemistry, Leiden Institute of Chemistry, Leiden University, Einsteinweg 55, 2300 RA Leiden, The Netherlands

${ }^{\S}$ Department of Chemistry, University of York, Heslington, York, YO10 5DD, U.K.

${ }^{\perp}$ Departament de Química Inorgànica i Orgànica (Secció de Química Orgànica) \& Institut de Quimica Teòrica i Computacional (IQTCUB), Universitat de Barcelona, Martí i Franquès 1, 08028 Barcelona, Spain

"Institució Catalana de Recerca i Estudis Avançats (ICREA), 08020 Barcelona, Spain

\section{Supporting Information}

ABSTRACT: The conformational analysis of glycosidases affords a route to their specific inhibition through transition-state mimicry. Inspired by the rapid reaction rates of cyclophellitol and cyclophellitol aziridine-both covalent retaining $\beta$-glucosidase inhibitors-we postulated that the corresponding carba "cyclopropyl" analogue would be a potent retaining $\beta$-glucosidase inhibitor for those enzymes reacting through the ${ }^{4} \mathrm{H}_{3}$ transition-state conformation. $A b$ initio metadynamics simulations of the conformational free energy landscape for the cyclopropyl inhibitors show a strong bias for the ${ }^{4} \mathrm{H}_{3}$ conformation, and carba-cyclophellitol, with an $\mathrm{N}$-(4-azidobutyl)carboxamide moiety, proved to be a potent inhibitor $\left(K_{\mathrm{i}}\right.$ $=8.2 \mathrm{nM})$ of the Thermotoga maritima TmGH1 $\beta$ glucosidase. 3-D structural analysis and comparison with unreacted epoxides show that this compound indeed binds in the ${ }^{4} \mathrm{H}_{3}$ conformation, suggesting that conformational strain induced through a cyclopropyl unit may add to the armory of tight-binding inhibitor designs.

$\mathrm{T}^{\mathrm{t}}$ he diverse conformational pathways of glycosidases ${ }^{1,2}$ (for example, Figure 1A) coupled to their phenomenal transition-state stabilization ${ }^{3}$ offer a powerful route to selective enzyme inhibition. One of the main goals of the field-very rarely achieved-is to design and apply conformationally restricted inhibitors in order to provide both potency and specificity; conformationally biased inhibitors that target specific classes of glycoside hydrolase $(\mathrm{GH})$ would be of considerable use as cellular and mechanistic probes with potential as starting points for therapeutic compounds. Cyclophellitol (1, Figure 1), isolated in 1990 from the mushroom Phellinus sp., ${ }^{4}$ is a potent mechanism-based inhibitor of retaining $\beta$-glucosidases. It finds primary use as a covalent inactivator of $\beta$-glucosidases. ${ }^{5}$ Cyclophellitol is a configurational analogue of $\beta$-glucopyranose, but its conformational behavior is different. Whereas $\beta$-glucopyranoses prefer to adopt a ${ }^{4} \mathrm{C}_{1}$ conformation, the epoxide annulation in 1 likely enforces a preferred ${ }^{4} \mathrm{H}_{3}$ half-chair conformation onto the cyclitol moiety.

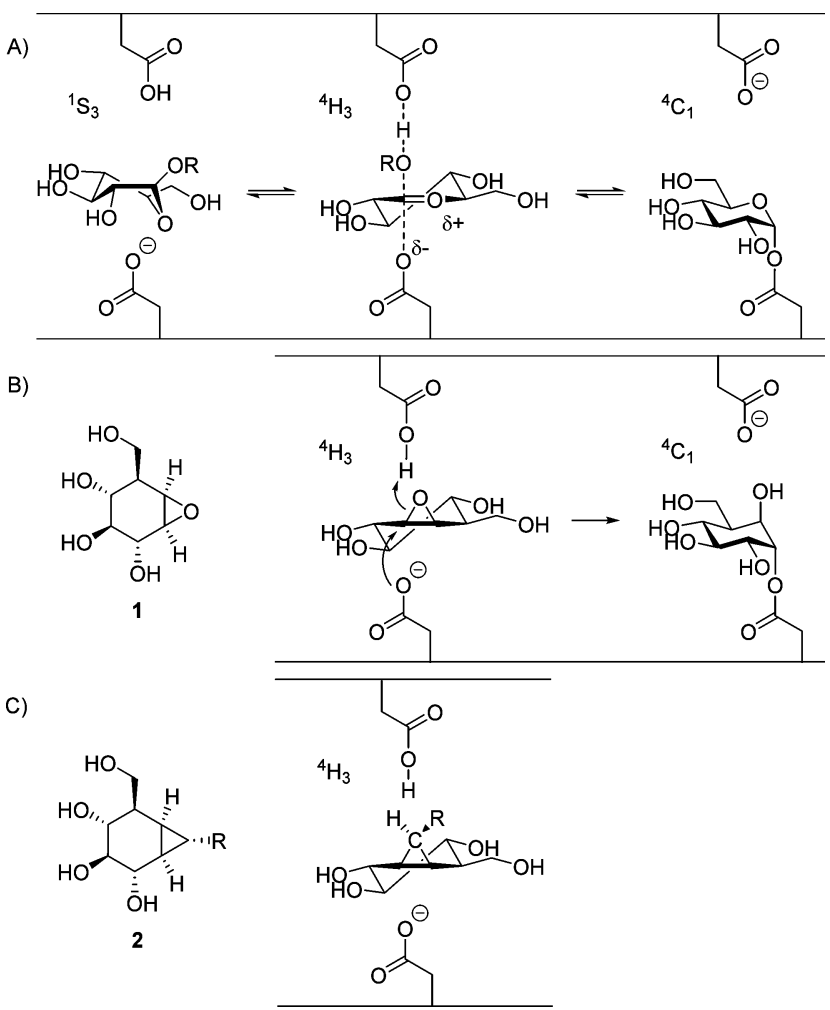

Figure 1. (A) Mechanistic itinerary of retaining $\beta$-glucosidases. (B) Structure of cyclophellitol (1) adopting a ${ }^{4} \mathrm{H}_{3}$ conformation and its proposed mechanism of binding. (C) Structure of carba-cyclophellitol (2) in ${ }^{4} \mathrm{H}_{3}$ conformation.

Cyclophellitol (1) is thus a potential conformational analogue of the oxocarbenium ion transition-state during $\beta$-glucosidasemediated hydrolysis of a $\beta$-glucosidic linkage.

Although the mode of action of $\mathbf{1}$ is covalent (Figure 1B), its potency and specificity as a retaining $\beta$-glucosidase inhibitor

Received: February 24, 2017

Published: May 2, 2017 
and its mode of action (entering the enzyme active site as a ${ }^{4} \mathrm{H}_{3}$ half-chair transition-state analogue followed by $\mathrm{S}_{\mathrm{N}} 2$ displacement of the epoxide heteroatom) led us to consider whether the corresponding carba analogue (that is, substitution of the oxygen for carbon) would result in competitive inhibitors in which potency and potentially specificity would be accrued by virtue of partial transition-state mimicry (Figure 1C).

To test this hypothesis, a set of carba-cyclophellitols was designed. Here we present the synthesis of carba-cyclophellitols 3-5 (Figure 2), the quantum mechanical analysis of their

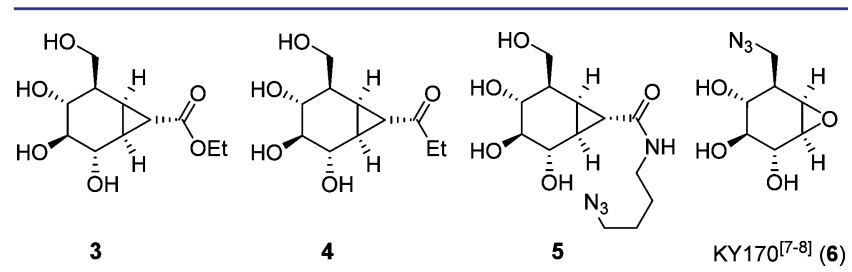

Figure 2. Structures of carba-cyclophellitols 3-5 and 8-azidocyclophellitol $\left(6, \mathrm{KY} 170^{7,8}\right)$.

favored conformation, and their structural and inhibitory dissection toward $\beta$-glucosidases. Carba-cyclophellitols are shown to be low $\mu \mathrm{M}$ inhibitors. Furthermore, exploiting the possibility of incorporating pseudoaxial $\mathrm{R}$ groups-consistent with the catalytic itinerary - that bearing a hydrophobic moiety at the terminal cyclopropyl carbon (5) was indeed a potent (low $\mathrm{nM}$ ) inhibitor of a classical model $\beta$-glucosidase, namely Thermotoga maritima TmGH1. ${ }^{5,6}$ The crystal structure of TmGH1 containing carba-cyclophellitol $\mathbf{5}$ was determined and compared with that of an unreacted cyclophellitol derivative; as predicted, both bind in ${ }^{4} \mathrm{H}_{3}$ conformation, which is the presumed transition-state conformation during the TmGH1catalyzed hydrolysis of $\beta$-glucosidic linkages.

The synthesis of compounds $\mathbf{3} \mathbf{- 5}$ commenced with the easy access of key intermediate 7 , which was obtained via the synthetic procedure described by the group of Madsen ${ }^{9}$ and optimized in our laboratory (Scheme 1$).^{8}$ Global benzylation of 7 gave cyclohexene 8 , and cyclopropanation with ethyl diazoacetate $(\mathrm{EDA})^{10,11}$ under the agency of $\mathrm{Cu}(\mathrm{acac})_{2}$ resulted in the formation of product 9 as a mixture of $\alpha$ - and $\beta$-isomers $(\alpha / \beta, 2: 1)$. After the reduction step ${ }^{12}$ the $\beta$-isomer could be isolated by column chromatography to give alcohol 10, which was oxidized, and ensuing esterification yielded enantiomerically pure $\beta$-ester 11. Sequential one-pot formation and Grignard addition onto the Weinreb amide yielded $\beta$-ketone 12. Both benzyl-protected ester 11 and ketone $\mathbf{1 2}$ were subjected to palladium-catalyzed hydrogenolysis conditions in ethyl acetate and acetic acid (11) or in methanol (12) to obtain target compounds 3 and 4 . The mixture of $\alpha$ - and $\beta$-esters 9 was saponified, and the resulting carboxylates were condensed with 4-azidobutan-1-amine (see Supporting Information (SI)). The mixture of $\alpha$ - and $\beta$-amides was separated by preparative HPLC purification. Finally, the benzyl groups were removed in the presence of the azide with anhydrous $\mathrm{BCl}_{3}$ in dichloromethane to afford $\beta$-amide $\mathbf{5}$.

Having carba-cyclopropane $\mathbf{3 - 5}$ in hand, we studied their inhibition potency in comparison with deoxynojirimycin (DNJ), a known competitive TmGH1 inhibitor and AMPDNM (MZ-21), a known human retaining $\beta$-glucosidase inhibitor. ${ }^{13}$ Initial binding constant $\left(K_{\mathrm{i}}\right)$ values were determined on $\mathrm{Tm} \mathrm{GH} 1$ by monitoring the UV absorbance of $p$ nitrophenolate from $p$-nitrophenyl $\beta$-D-glucopyranoside using
Scheme 1. The synthesis of carba-cyclophellitols $3-5^{a}$

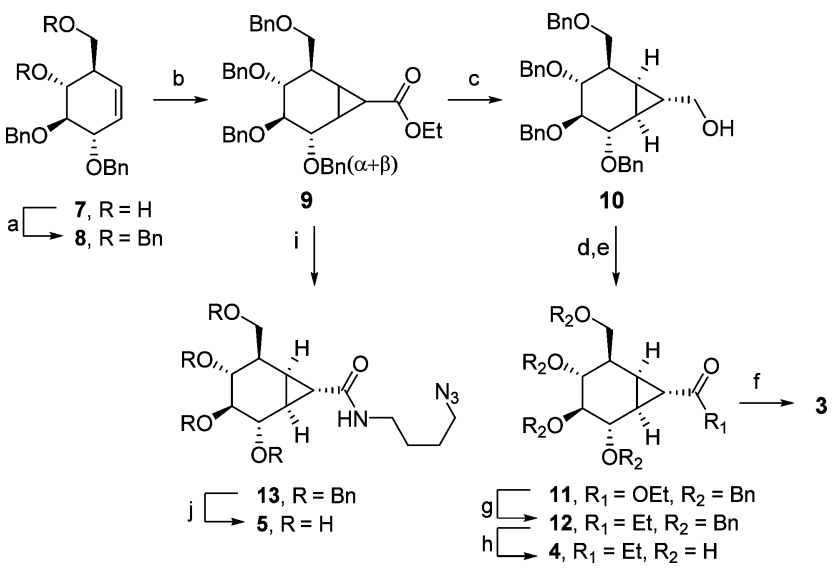

${ }^{a}$ Reagents and conditions: (a) BnBr, NaH, TBAI, DMF, $0{ }^{\circ} \mathrm{C}$ to rt, 24 h, 94\%; (b) EDA, $\mathrm{Cu}(\mathrm{acac})_{2}$, EtOAc, $(35 \%, 2: 1$, as a mixture of $\alpha / \beta)$; (c) DIBAL, THF, $30 \mathrm{~min}$ at $0{ }^{\circ} \mathrm{C}$ and then $1 \mathrm{~h}$ at rt, $13 \%$; (d) Jones reagent, acetone, $0{ }^{\circ} \mathrm{C}, 3 \mathrm{~h}, 53 \%$; (e) EtOH, $N, N^{\prime}$-diisopropylcarbodiimide, 4-dimethylaminopyridine, toluene, rt, 4 h, $62 \%$; (f) $\mathrm{Pd}(\mathrm{OH})_{2} /$ $\mathrm{C}, \mathrm{H}_{2}$, EtOAc, AcOH, rt, overnight, 81\%; (g) N,O-dimethylhydroxylamine hydrochloride, EtMgBr, THF, 48\%; (h) $\mathrm{Pd}(\mathrm{OH})_{2} / \mathrm{C}, \mathrm{H}_{2}$, $\mathrm{MeOH}$, rt, overnight, (58\%); (i) i) $\mathrm{LiOH}, \mathrm{MeOH}, \mathrm{H}_{2} \mathrm{O}$, rt, overnight; ii) 4-azidobutan-1-amine (see SI), DIPEA, HCTU, $\mathrm{CH}_{2} \mathrm{Cl}_{2}, \mathrm{rt}$, overnight; (j) $\mathrm{BCl}_{3}, \mathrm{DCM}, 99 \%$.

the Lineweaver-Burk method. Carba-cyclophellitol 3 and 4 showed micromolar inhibition, consistent with our design strategy and similar to that displayed by the charged species DNJ, whereas 5 proved to be a strong reversible binding Tm $\mathrm{GH} 1$ inhibitor with a $K_{\mathrm{i}}$ value of $8.2 \mathrm{nM}$, much more potent than $\mathrm{DNJ}^{14}$ and AMP-DNM (low micromolar) (Table 1 and

Table 1. Apparent $\mathrm{IC}_{50}$ Values and Inhibitory Constants $\left(K_{\mathrm{i}}\right)$ for in Vitro Inhibition of $\alpha$ - and $\beta$-Glucosidase Activity by Compounds 3-5, DNJ, and AMP-DNM

\begin{tabular}{|c|c|c|c|}
\hline \multirow[b]{2}{*}{ compound } & \multirow{2}{*}{$\frac{K_{\mathrm{i}}^{a}}{T m \mathrm{GH} 1^{b}}$} & \multicolumn{2}{|c|}{ app $\mathrm{IC}_{50}$} \\
\hline & & $\mathrm{GBAl}^{c}$ & $\mathrm{GAA}^{c}$ \\
\hline 3 & $22.3 \mu \mathrm{M}$ & $>150 \mu \mathrm{M}$ & $>150 \mu \mathrm{M}$ \\
\hline 4 & $88.9 \mu \mathrm{M}$ & $>150 \mu \mathrm{M}$ & $>150 \mu \mathrm{M}$ \\
\hline 5 & $8.20 \mathrm{nM}$ & $99 \pm 1.9 \mu \mathrm{M}$ & $>150 \mu \mathrm{M}$ \\
\hline DNJ & $2.50 \mu \mathrm{M}^{d}$ & $109 \pm 1.0 \mu \mathrm{M}^{e}$ & $1.5 \mu \mathrm{M}^{g}$ \\
\hline AMP-DNM (MZ-21) & $4.97 \mu \mathrm{M}$ & $156 \pm 16 \mathrm{nM}^{f}$ & $0.4 \mu \mathrm{M}^{g}$ \\
\hline
\end{tabular}

${ }^{a} K_{\mathrm{m}}$ Tm GH1 $=0.24 \mathrm{mM} .{ }^{b}$ The assay was performed with $p$-NPG as substrate. ${ }^{c}$ The assay was performed with 2,4-DNPG as substrate. Values in agreement with literature. ${ }^{d} K_{\mathrm{i}} \mathrm{DNJ}=3.8 \mu \mathrm{M}$ in TmGH1. ${ }^{14}$ ${ }^{e} \mathrm{IC}_{50} \mathrm{DNJ}=250 \mu \mathrm{M}$ in GBA1. ${ }^{15} \mathrm{fC}_{50}$ AMP-DNM $=100-200 \mathrm{nM}$ in GBA1. ${ }^{15,16} g$ Values from ref 17 . App: apparent.

Figure S4). We then explored the activity of compound 5 in human lysosomal retaining $\beta$-glucosidase, GBA1 (deficiency of which is causative of the human lysosomal storage disorder, Gaucher disease) with an apparent $\mathrm{IC}_{50} \approx 100 \mu \mathrm{M}$. No apparent inhibition of the human lysosomal $\alpha$-glucosidase, GAA (deficient in the human glycogen storage disease, Pompe disease) was observed at final concentrations of 5 up to 150 $\mu \mathrm{M}$. Thus, although less potent for GBA1 than for the bacterial enzyme tested, compound 5 appears to have selectivity for the human lysosomal $\beta$-glucosidase over the human lysosomal $\alpha$ glucosidase, which is opposite of the selectivity observed for DNJ (Table 1). 
Inspired by the low $\mu \mathrm{M}$ to $\mathrm{nM}$ inhibition of $T m \mathrm{GH} 1$ by the carba-cyclopropanes, we sought to determine whether the cyclopropyl moiety indeed biased the conformation to ${ }^{4} \mathrm{H}_{3}$. We calculated the conformational free energy landscape (FEL) for generic cyclopropyl $(2, \mathrm{R}=\mathrm{H})$ by ab initio metadynamics (see SI), and the Cremer-Pople puckering coordinates $\theta$ and $\phi$ were used as collective variables, yielding a Mercator representation for the FEL (as used previously for diverse glycosidase inhibitors ${ }^{18-20}$ ), Figure 3 A. Compound 2 clearly

A)

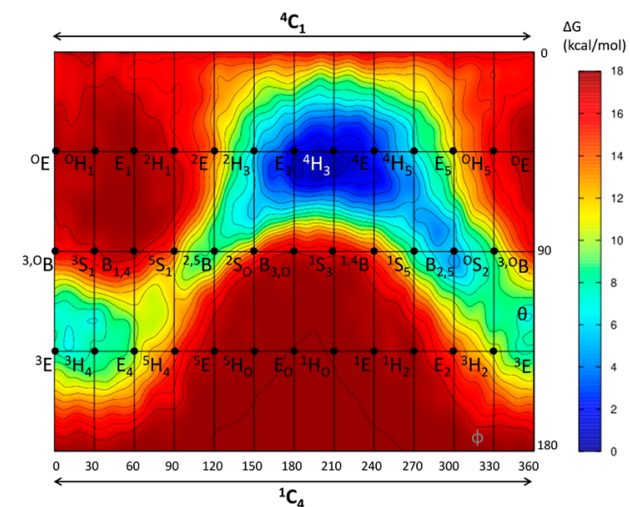

B)

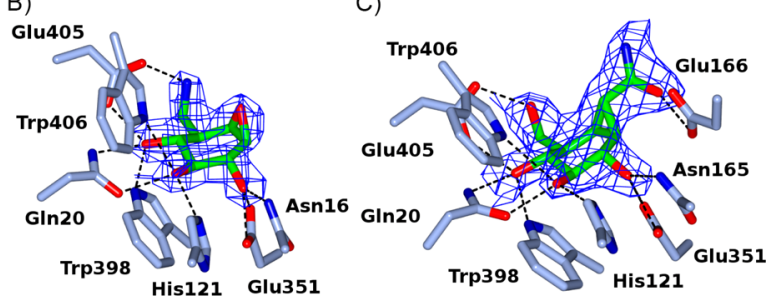

D)

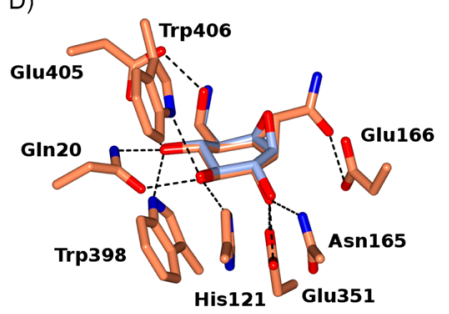

Figure 3. (A) A mercator representation for the computed free energy landscape (FEL) of cyclopropyl $(2, \mathrm{R}=\mathrm{H})(\theta$ and $\phi$ are given in degrees). (B) Crystal structure of $\mathrm{TmGH} 1$ in complex with unreacted 6, KY170. (C) Crystal structure of TmGH1 in complex with carbacyclophellitol 5, showing the carba-cyclophellitol CO-NH group. Electron density maps for both (B) and (C) are maximum likelihood/ $\sigma_{\mathrm{A}}$ weighted $2 F_{\mathrm{obs}}-F_{\text {calc }}$ syntheses contoured at $1.4 \sigma$. (D) Overlay of (B) in ice blue on (C) in coral.

favors the ${ }^{4} \mathrm{H}_{3}$ conformation in vacuo, with the flipped ${ }^{3} \mathrm{H}_{4}$ form in another local energy minimum. Subsequent to FEL calculation, we compared the experimental $J$ values of several (cyclohexane) ring protons of compound 4 with their calculated counterparts, in which calculations were performed on compound 4 in the ${ }^{4} \mathrm{H}_{3}$ conformation. Both sets of values are in good agreement, which underscores the notion that compound 4, and by extension also the other compounds subject of this Communication (whose ${ }^{1} \mathrm{H}$ NMR spectra give broadened signals due to the amide present-see SI) do indeed adopt the ${ }^{4} \mathrm{H}_{3}$ conformation in solution.
Structural dissection of the inhibitory action of $\mathbf{5}$, and the conceptual link through to cyclophellitol 1, was achieved first by rapid soaking (as opposed to preincubation as used previously to trap the covalent adduct ${ }^{5}$ ) of crystals of Tm GH1 with cyclophellitol derivative $\mathrm{KY} 170^{7,8}$ (6). Serendipitously, this indeed afforded the unreacted cyclophellitol KY170 in ${ }^{4} \mathrm{H}_{3}$ conformation, with the nucleophile poised to attack, Figure 3B, confirming our hypothesis that (unreacted) cyclophellitols adopt a transition-state like ${ }^{4} \mathrm{H}_{3}$ conformation. In order to dissect similar mimicry by carba-cyclopropane $\mathbf{5}$, and confirm the FEL calculated by $a b$ initio metadynamics, TmGH1 crystals were soaked with carba-cyclophellitol $\mathbf{5}$ and the subsequently obtained structure was analyzed and solved with X-ray crystallography. The obtained electron density pattern clearly demonstrates the presence of carba-cyclophellitol 5 in the active site in ${ }^{4} \mathrm{H}_{3}$ conformation (Figure $3 \mathrm{C}$; the butyl azide moiety is mobile and differently disordered in the structure and not shown for clarity).

Overlay of cyclophellitol derivative KY170 with carbacyclophellitol 5 (Figure 3D) shows almost perfect coincidence of atomic positions, showing that, as suggested by the FEL, $\mathbf{5}$ is a permanent mimic of cyclophellitol posted in the active site prior to nucleophilic attack.

The improved binding of $\mathbf{5}$, relative to $\mathbf{3}$ and $\mathbf{4}$ presumably stems from desolvation caused by the alkyl-azido "tail" sitting in the aglycone site. One of the design advantages of the carbacyclopropanes is that any pendent $\mathrm{R}$ groups are disposed pseudoaxial to the sugar ring, consistent with the distortions seen during catalysis which presumably adds to their augmentation of binding. The 3-D structure with 5 confirms this and shows a lateral, antitrajectory interaction of the catalytic amino acid Glu166 with the pseudoaxially disposed amide of 5. There are four molecules of $\mathrm{Tm} \mathrm{GH} 1$ in the crystallographically observed asymmetric unit. While they all show the $\mathrm{R}$ group axial, they all show different degrees of disorder of this alkyl region itself. In one molecule, there is essentially no electron density for the tail, while in two molecules the chain passes through the aglycon region (that is flanked by Val169, Trp168, and Trp324), making nonspecific interactions with this region. In the fourth molecule of the $\mathrm{AU}$, the alkyl azido chain appears to follow two separate routes along each hydrophobic flank of the substrate binding cleft.

Bicyclic cyclopropyl glucosidase inhibitors, with the bridge between the "C6" and "O5" atoms, were first proposed by Tanaka and co-workers ${ }^{21}$ and later developed in galacto configuration by Bennet and co-workers and found to be good $\alpha$-glucosidase and galactosidase inhibitors, respectively. ${ }^{22}$ More recently, activated forms of these compounds have been used as covalent inhibitors. ${ }^{23}$ In these cases the conformational restriction limits the accessible conformations to "off-pathway" ${ }^{3} \mathrm{H}_{2}$ and ${ }^{2} \mathrm{H}_{3}$ half-chairs ${ }^{23}$ (or perhaps their related 1,4 boats) recently elegantly revealed by X-ray crystallography. ${ }^{24}$ Further, Stick and Stubbs ${ }^{25}$ synthesized a bicyclic cyclopropyl inhibitor with the bridge between the "anomeric" $\mathrm{Cl}$ carbon position and the "C2" atom with a millimolar $K_{\mathrm{i}}$ value. The carbacyclophellitol derivatives presented here offer, by virtue of the advantage of their conformational restriction between the "O5" and "anomeric" C1 carbon positions, a potent inhibitor in which the conformational restraint is a glycosidase reaction coordinate relevant ${ }^{4} \mathrm{H}_{3}$. Given the large number of glycosidase inhibitors in medical use, including those being developed as pharmacological chaperones and as diagnostic tools, the harnessing of appropriate conformation restraint, coupled to 
correct stereochemistry, should add greatly to the enzymological, cellular, and, ultimately, therapeutic toolbox.

\section{ASSOCIATED CONTENT}

\section{S Supporting Information}

The Supporting Information is available free of charge on the ACS Publications website at DOI: 10.1021/jacs.7b01773.

Primary NMR data files for 3-5, 8, 10-14 (ZIP)

Experimental procedures, Figures $\mathrm{S} 1-\mathrm{S} 5$ and Table S1, and ${ }^{1} \mathrm{H}$ and ${ }^{13} \mathrm{C}$ NMR spectra (PDF)

S

\section{AUTHOR INFORMATION}

\section{Corresponding Authors}

*h.s.overkleeft@lic.leidenuniv.nl

*gideon.davies@york.ac.uk

\section{ORCID}

Dennis P. A. Wander: 0000-0003-3881-5240

Jeroen D. C. Codée: 0000-0003-3531-2138

Carme Rovira: 0000-0003-1477-5010

Gideon J. Davies: 0000-0002-7343-776X

Herman S. Overkleeft: 0000-0001-6976-7005

Notes

The authors declare no competing financial interest.

\section{ACKNOWLEDGMENTS}

We thank The Netherlands Organization for Scientific Research (NWO-CW, ChemThem grant to J.M.A. and H.S.O.), the European Research Council (ERC-2011-AdG290836 "Chembiosphing" to H.S.O., and ERC-2012-AdG32294 "Glycopoise" to G.J.D.), the Spanish Ministry of Economy and Competitiveness (CTQ2014-55174-P to C.R.), and the Generalitat de Catalunya (2014SGR-987 to C.R.) for financial support. L.R. thanks the University of Barcelona for an APIF predoctoral fellowship. We gratefully acknowledge the computer resources at MareNostrum and the technical support provided by BSC-CNS (RES-QCM-2016-3-00017). We thank the European Synchrotron Radiation Facility at Grenoble for access to beamline ID23-2 and the Diamond Light Source for access to beamline IO2 (proposal no. mx-13587) that contributed to the results presented here.

\section{REFERENCES}

(1) Davies, G. J.; Planas, A.; Rovira, C. Acc. Chem. Res. 2012, 45, 308.

(2) Speciale, G.; Thompson, A. J.; Davies, G. J.; Williams, S. J. Curr. Opin. Struct. Biol. 2014, 28, 1.

(3) Wolfenden, R.; Snider, M. J. Acc. Chem. Res. 2001, 34, 938.

(4) Atsumi, S.; Umezawa, K.; Iinuma, H.; Naganawa, H.; Nakamura, H.; litaka, Y.; Takeuchi, T. J. Antibiot. 1990, 43, 49.

(5) Gloster, T. M.; Madsen, R.; Davies, G. J. Org. Biomol. Chem. 2007, 5, 444.

(6) Gloster, T. M.; Meloncelli, P.; Stick, R. V.; Zechel, D.; Vasella, A.; Davies, G. J. J. Am. Chem. Soc. 2007, 129, 2345.

(7) Witte, M. D.; Kallemeijn, W. W.; Aten, J.; Li, K.-Y.; Strijland, A.; Donker-Koopman, W. E.; van den Nieuwendijk, A. M. C. H.; Bleijlevens, B.; Kramer, G.; Florea, B. I.; Hooibrink, B.; Hollak, C. E. M.; Ottenhoff, R.; Boot, R. G.; van der Marel, G. A.; Overkleeft, H. S.; Aerts, J. M. F. G. Nat. Chem. Biol. 2010, 6, 907.

(8) Li, K.-Y.; Jiang, J.; Witte, M. D.; Kallemeijn, W. W.; van den Elst, H.; Wong, C.-S.; Chander, S. D.; Hoogendoorn, S.; Beenakker, T. J. M.; Codée, J. D. C.; Aerts, J. M. F. G.; van der Marel, G. A.; Overkleeft, H. S. Eur. J. Org. Chem. 2014, 2014, 6030.
(9) Hansen, F. G.; Bundgaard, E.; Madsen, R. J. Org. Chem. 2005, 70, 10139.

(10) Ye, T.; McKervey, M. A. Chem. Rev. 1994, 94, 1091.

(11) Caballero, A.; Prieto, A.; Diaz-Requejo, M. M.; Pérez, P. J. Eur. J. Inorg. Chem. 2009, 2009, 1137.

(12) Zhou, S.; Kern, E. R.; Gullen, E.; Cheng, Y.-C.; Drach, J. C.; Tamiya, S.; Mitsuya, H.; Zemlicka, J. J. Med. Chem. 2006, 49, 6120.

(13) Overkleeft, H. S.; Renkema, G. H.; Neele, J.; Vianello, P.; Hung, I. O.; Strijland, A.; van der Burg, A. M.; Koomen, G. J.; Pandit, U. K.; Aerts, J. M. J. Biol. Chem. 1998, 273, 26522.

(14) Zechel, D. L.; Boraston, A. B.; Gloster, T.; Boraston, C. M.; Macdonald, J. M.; Tilbrook, D. M. G.; Stick, R. V.; Davies, G. J. J. Am. Chem. Soc. 2003, 125, 14313.

(15) Wennekes, T.; Meijer, A. J.; Groen, A. K.; Boot, R. G.; Groener, J. E.; van Eijk, M.; Ottenhoff, R.; Bijl, N.; Ghauharali, K.; Song, H.; O'Shea, T. J.; Liu, H.; Yew, N.; Copeland, D.; van den Berg, R. J.; van der Marel, G. A.; Overkleeft, H. S.; Aerts, J. M. J. Med. Chem. 2010, 53, 689.

(16) Ghisaidoobe, A. T.; van den Berg, R. J. B. H. N.; Butt, S. S.; Strijland, A.; Donker-Koopman, W. E.; Scheij, S.; van den Nieuwendijk, A. M. C. H.; Koomen, G.-J.; van Loevezijn, A.; Leemhuis, M.; Wennekes, T.; van der Stelt, M.; van der Marel, G. A.; van Boeckel, C. A. A.; Aerts, J. M. F. G.; Overkleeft, H. S. J. Med. Chem. 2014, 57, 9096.

(17) Wennekes, T.; van den Berg, R. J. B. H. N.; Donker, W.; van der Marel, G. A.; Strijland, A.; Aerts, J. M. F. G.; Overkleeft, H. S. J. Org. Chem. 2007, 72, 1088.

(18) Petricevic, M.; Sobala, L. F.; Fernandes, P. Z.; Raich, L.; Thompson, A. J.; Bernardo-Seisdedos, G.; Millet, O.; Zhu, S.; Sollogoub, M.; Jiménez-Barbero, J.; Rovira, C.; Davies, G. J.; Williams, S. J. J. Am. Chem. Soc. 2017, 139, 1089.

(19) Thompson, A. J.; Dabin, J.; Iglesias-Fernández, J.; Ardèvol, A.; Dinev, Z.; Williams, S. J.; Bande, O.; Siriwardena, A.; Moreland, C.; Hu, T.-C.; Smith, D. K.; Gilbert, H. J.; Rovira, C.; Davies, G. J. Angew. Chem., Int. Ed. 2012, 51, 10997.

(20) Williams, R. J.; Iglesias-Fernández, J.; Stepper, J.; Jackson, A.; Thompson, A. J.; Lowe, E. C.; White, J. M.; Gilbert, H. J.; Rovira, C.; Davies, G. J.; Williams, S. J. Angew. Chem., Int. Ed. 2014, 53, 1087.

(21) Tanaka, K. S. E.; Winters, G. C.; Batchelor, R. J.; Einstein, F. W. B.; Bennet, A. J. J. Am. Chem. Soc. 2001, 123, 998.

(22) Wang, Y.; Bennet, A. J. Org. Biomol. Chem. 2007, 5, 1731.

(23) Chakladar, S.; Wang, Y.; Clark, T.; Cheng, L.; Ko, S.; Vocadlo, D. J.; Bennet, A. J. Nat. Commun. 2014, 5, 5590.

(24) Adamson, C.; Pengelly, R. J.; Shamsi Kazem Abadi, S.; Chakladar, S.; Draper, J.; Britton, R.; Gloster, T. M.; Bennet, A. J. Angew. Chem. 2016, 128, 15202.

(25) Stick, R. V.; Stubbs, K. A. J. Carbohydr. Chem. 2005, 24, 529. 